\title{
Fundamental parameters of the highly reddened young open clusters Westerlund 1 and $2^{\star, \star \star}$
}

\author{
A.E. Piatti ${ }^{1}$, E. Bica ${ }^{2}$, and J.J. Clariá ${ }^{1}$ \\ 1 Observatorio Astronómico, Universidad Nacional de Córdoba, Laprida 854, 5000 Córdoba, Argentina \\ 2 Instituto de Física, Universidade Federal do Rio Grande do Sul, Av. Bento Gonçalves, 9500, Caixa Postal 15051, CEP \\ 91501-970, Porto Alegre, RS, Brazil
}

Received April 2; accepted May 23, 1997

\begin{abstract}
We study the compact open clusters Westerlund 1 (BH 197) and Westerlund 2. We present CCD integrated spectroscopy for both clusters, and CCD imaging in the $V$ and $I$ bands for the former one. So far, Westerlund 1 is possibly the most reddened open cluster studied in detail $\left(A_{v} \approx 13.0\right)$. It has an age of $8 \pm 3 \mathrm{Myr}$ and a distance from the Sun of $d_{\odot} \approx 1.0 \pm 0.4 \mathrm{kpc}$. For Westerlund 2 we derive a visual absorption $A_{V} \approx 5.0 \mathrm{mag}$, an age of $2-3 \mathrm{Myr}$, and $d_{\odot}=5.7 \pm 0.3 \mathrm{kpc}$. From luminosity and structural arguments we conclude that Westerlund 1, although young and compact, it is a massive cluster, in contrast to Westerlund 2.
\end{abstract}

Key words: open clusters: individual: Westerlund 1, Westerlund 2 - open clusters: general

\section{Introduction}

Since early studies (Gascoigne \& Kron 1952; Hodge 1961), blue star clusters with globular cluster appearance have been known to exist in the Magellanic Clouds, where they are a common phenomenon. Very few young disk clusters in our Galaxy can be considered as such. An example is NGC 3603, which is populous and has a compact core also known as HD 97950 (Moffat et al. 1985). It is located in

Send offprint requests to: A.E. Piatti, e-mail: andres@oac.uncor.edu

* Based on observations made at Complejo Astronómico El Leoncito, which is operated under agreement between the Consejo Nacional de Investigaciones Científicas y Técnicas de la República Argentina and the Universities of La Plata, Córdoba and San Juan, Argentina, and at the University of Toronto (David Dunlap Observatory) 24-inch telescope, Las Campanas, Chile.

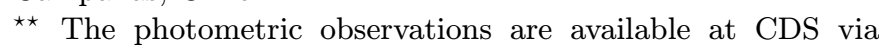
anonymous ftp to cdsarc.u-strasbg.fr (130.79.128.5) or via http://cdsweb.u-strasbg.fr/Abstract.html a giant HII region/molecular cloud complex (e.g. Moffat 1983; Melnick et al. 1989). The identification of other open clusters with similar characteristics in the Milky Way disk is of great interest, since they may provide important clues on the environmental and internal conditions for enhanced star formation processes.

Westerlund 1 was first detected by Westerlund (1961) using the Uppsala Southern Station Schmidt telescope in Australia. The cluster is heavily reddened and his early $V, I$ photographic photometry suggested $A_{V}=12.0 \mathrm{mag}$, an age of $3 \mathrm{Myr}$ and a distance of $1.4 \mathrm{kpc}$. In fact, in the blue, the cluster is virtually invisible, even in the deep SRC J Sky Survey Schmidt plate, being prominent in the ESO/SERC red Schmidt plate. Westerlund 1 is located in the southern Milky Way in Ara, at (B1950) $\alpha=16^{\mathrm{h}} 43^{\mathrm{m}}$ $12^{\mathrm{s}}, \delta=-45^{\mathrm{o}} 44^{\prime}$. The cluster, also known as OCl 985 in Alter et al. (1970), is projected not far from the central regions of the Galaxy $\left(\ell=339.56^{\circ}, b=-0.36^{\circ}\right)$. It was included with nominal coordinates in the ESO/Uppsala survey of the ESO (B) Atlas as ESO277-SC12 (Holmberg et al. 1977), since it was of course invisible in the blue plate. Westerlund 1 is $\mathrm{BH} 197$ in the Curtis-Schmidt star cluster survey of the southern Milky Way by van den Bergh \& Hagen (1975). Westerlund (1968) pointed out that the cluster could be identified as an extended source in the $2.2 \mu \mathrm{m}$ survey of Price (1968). Borgman et al. (1970) carried out photometry in the $K$ band of 12 stars in the region and concluded that several of them could be supergiant members. They estimated a visual absorption of at least $A_{v}=12.8 \mathrm{mag}$ for the cluster. More recently, Westerlund (1987, hereafter W87) using VRI photographic photometry for 258 stars together with nearinfrared spectroscopy of the brightest stars found $A_{v}=$ $10.0 \mathrm{mag}$ and derived a significantly larger distance of $5 \mathrm{kpc}$.

Westerlund 2 was first reported by Westerlund (1960). It is located in the southern Milky Way in Carina, at (B1950) $\alpha=10^{\mathrm{h}} 22^{\mathrm{m}} 12^{\mathrm{s}}, \delta=-57^{\mathrm{o}} 30$ ! 4 , which convert to $\ell=284.27^{\circ}, b=-0.33^{\circ}$. The cluster is also known 
as $\mathrm{OCl} 807, \mathrm{BH} 95$ and ESO127-SC18. Westerlund 2 is a tight open cluster, which is the core of the HII region RCW 49 (Belloni \& Mereghetti 1994, and references therein). Moffat et al. (1991, hereafter MSP91) carried out $U B V$ CCD photometry, estimating a distance of $7.9_{-1.0}^{+1.2} \mathrm{kpc}$. They also identified spectroscopically a WolfRayet member of WN 7 type. The earliest spectral type in the cluster is O $7 \mathrm{~V}$. In a comparison with NGC 3603, MSP91 concluded that Westerlund 2 is only slightly less massive and structurally much less concentrated.

In this paper, a CCD colour-magnitude diagram of Westerlund 1 is presented for the first time, in view of determining more accurately the cluster parameters. We also present CCD integrated spectroscopy for both Westerlund 1 and 2, and derive cluster parameters by comparing their properties with those of Galactic and Large Magellanic Cloud clusters (Bica 1988; Bica et al. 1990; Santos Jr. \& Bica 1993; Santos Jr. et al. 1995). In Sect. 2 we describe the photometric and spectroscopic observations and their calibrations. In Sect. 3 we present a $V$ vs. $(V-I)$ colour-magnitude diagram (CMD) of Westerlund 1 and discuss its properties. In Sect. 4 we analyse the CCD integrated spectra of Westerlund 1 and Westerlund 2. In Sect. 5 we carry out a simple structural analysis of Westerlund 1 in order to check its similarity to NGC 3603. Finally, in Sect. 6 we present the main conclusions of this work.

\section{Observations and reductions}

We carried out observations of stars in the region of the open cluster Westerlund 1 through the $V$ and $I$ passbands during a photometric night on July $2^{\text {nd }} 1995$. The direct images were obtained with the 24-inch telescope of the University of Toronto Southern Observatory situated at Las Campanas Observatory, Chile, equipped with a PM $512 \times 512$ METACHROME CCD coated to give improved blue response. The scale on the chip is 0 "' 45 per pixel, and consequently the sky area covered by a single frame is about $4^{\prime} \times 4^{\prime}$.

Table 1 summarizes the observational data, while $V$ and $I$ images of Westerlund 1 are provided in Figs. 1a and $1 \mathrm{~b}$, respectively. Notice that the $I$ image is much deeper even when obtained with an exposure time a factor thirty shorter than that of the $V$ image, because of the smaller reddening effects.

Observations of twelve standard stars located in the fields SA 107, SA 108 and SA 112 (Landolt 1992) were obtained in each filter at the beginning, middle, and end of the night, respectively. In addition, a series of bias exposures, flat-field frames on the twilight sky and dome were taken.

The observations have been reduced using the facilities at the Observatorio Astronómico of the National University of Córdoba. In order to clean the images by removing their instrumental signatures, we have applied
Table 1. Journal of the CCD imaging

\begin{tabular}{lllll}
\hline $\begin{array}{l}\text { Date } \\
\text { (UT) }\end{array}$ & $\begin{array}{l}\text { Time } \\
(\mathrm{UT})\end{array}$ & Filter & $\begin{array}{l}\text { Exposure } \\
(\text { secs })\end{array}$ & $\begin{array}{l}\text { FWHM } \\
\left({ }^{\prime \prime}\right)\end{array}$ \\
\hline July 2, 1995 & $4^{\mathrm{hs}} 23^{\mathrm{min}}$ & $V$ & 600 & 1.3 \\
July 2, 1995 & $4^{\mathrm{hs}} 34^{\mathrm{min}}$ & $V$ & 900 & 1.4 \\
July 2, 1995 & $4^{\mathrm{hs}} 50^{\mathrm{min}}$ & $I$ & 10 & 1.4 \\
July 2, 1995 & $4^{\mathrm{hs}} 52^{\mathrm{min}}$ & $I$ & 30 & 1.5 \\
July 2, 1995 & $4^{\mathrm{hs}} 54^{\text {min }}$ & $I$ & 30 & 1.5 \\
\hline
\end{tabular}

the averaged bias and flat-field corrections with IRAF standard routines. The average twilight and dome flatfields were also used to check possible effects due to nonperpendicularity of the optical path on the chip; no correction was necessary.

To obtain instrumental $v$ and $i$ magnitudes, we have used the DAOPHOT (Stetson 1991) package in the standard way on the IRAF environment. Aperture photometry of standard stars was performed with an aperture radius equal to 16 pixels $(7 ! ' 2)$. The following relations between the instrumental (lower case letters) and standard (capital letters) colours and magnitudes were adopted:

$v=6.71( \pm 0.09)+V+0.08( \pm 0.08) X_{v}-0.05( \pm 0.01)(V-I)$

$$
\begin{aligned}
i=6.34( \pm 0.11)+V-(V-I) & -0.04( \pm 0.10) X_{i} \\
& -0.07( \pm 0.01)(V-I)
\end{aligned}
$$

where $X_{v}, X_{i}$ are the airmasses corresponding to the observations in the $V$ and $I$ filters, respectively. The rms error affecting the calibration in Eq. (1) is $0.009 \mathrm{mag}$, while the corresponding one in Eq. (2) is 0.011 mag. The inversion of these expressions allowed us to transform the instrumental colour and magnitudes of the stars measured in the field of Westerlund 1 to the standard VI JohnsonCousins system.

At this stage, we have obtained three independent $V,(V-I)$ table sets available upon request, together with the $\mathrm{V}$ frame coordinates $(X$ and $Y)$ and the instrumental DAOPHOT.ALLSTARS rms errors. Then, we performed a cross-correlation identification between all $V,(V-I)$ tables averaging $V$ and $(V-I)$ values after applying appropriate offsets to the coordinates. The results are an improved set of colour and magnitude values as compared to those based on a single measure. Consequently, for stars with two or three measures, we could minimize possible anomalous values caused by the presence of contamination (e.g., cosmic rays). This procedure also allowed us to estimate the photometric internal errors $(\sigma)$. Typically $\sigma V \lesssim 0.02 \mathrm{mag}$ and $\sigma(V-I) \lesssim 0.03 \mathrm{mag}$ for $V<15 \mathrm{mag}$, increasing to 0.04 and 0.05 at $V \approx 18$, respectively.

The integrated spectroscopic observations were carried out with the $2.15 \mathrm{~m}$ telescope at the Complejo 


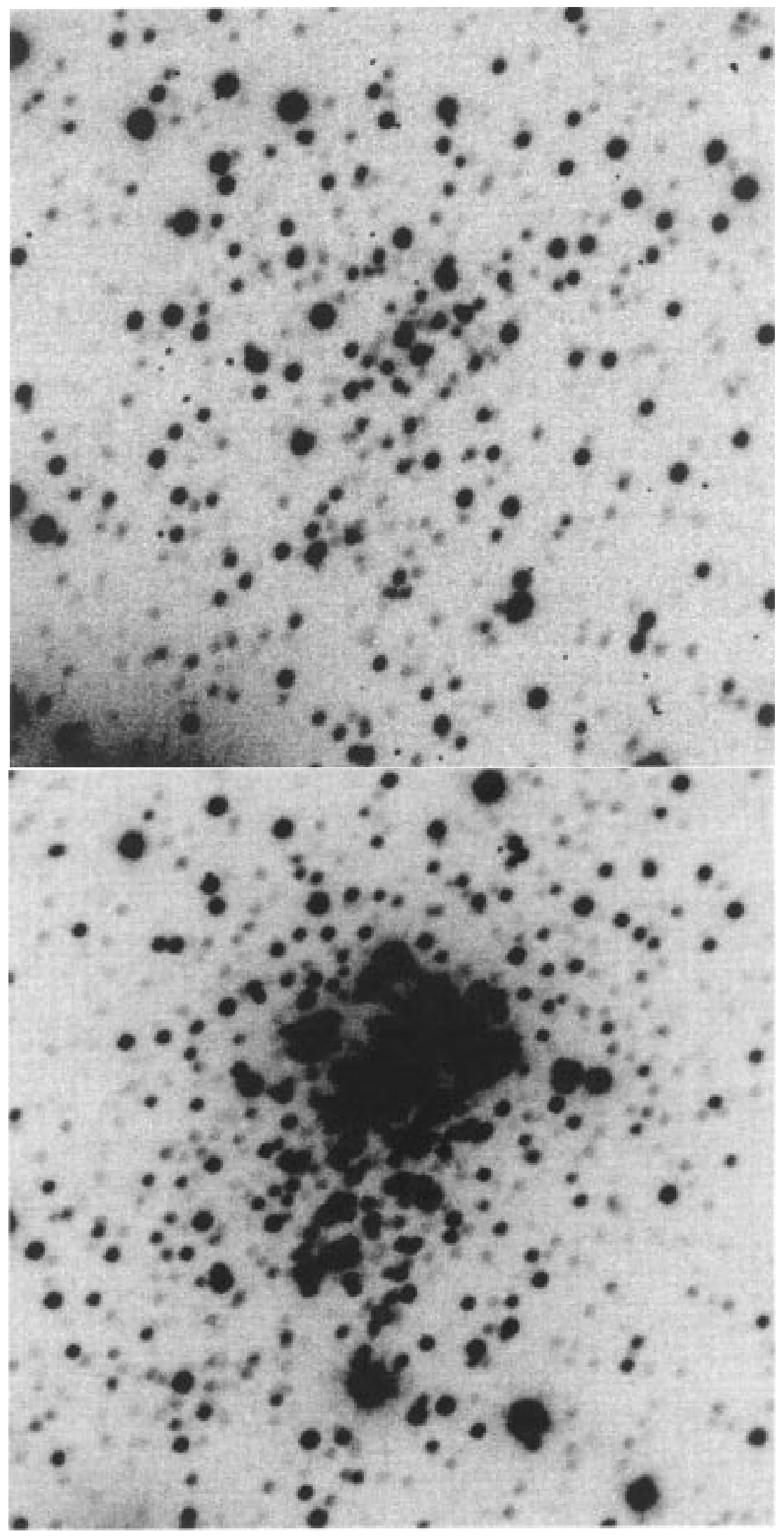

Fig. 1. Image of Westerlund 1 (north is up and east is to the right). The field is $4^{\prime} \times 4^{\prime}:$ a) $V$ filter (900 s), b) $I$ filter (30 s) 
Astronómico El Leoncito (CASLEO, Argentina) during a run in May 1995. We employed a CCD camera containing a Tektronics chip of $1024 \times 1024$ pixels attached to a REOSC spectrograph, the size of each pixel being $24 \times 24 \mu \mathrm{m}$. The slit was oriented in the east-west direction and the observations were performed by scanning the slit across the objects in the north-south direction, in order to get a proper sampling of cluster stars. The total field along the slit was 4.7 , allowing us to sample background regions. We used a grating of 300 grooves $\mathrm{mm}^{-1}$ in two different set-ups, namely "blue nights" and "red nights": (a) during the blue nights we obtained spectra covering a range from 3500 to $7000 \AA$, with an average dispersion in the observed region of $\sim 140 \AA / \mathrm{mm}$ or $3.46 \AA /$ pixel. The slit width was 4 ". 2 resulting a mean resolution of $14 \AA$, according to the FWHM of the He-Ar comparison lamps. (b) In the red nights the range was $5800-9200 \AA$ with a similar dispersion $(3.36 \AA /$ pixel $)$. The slit width was 4.2 resulting a mean resolution of $\approx 17 \AA$. An OG 550 filter was employed to eliminate the second order contamination.

A series of exposure of 15 minutes each was employed for Westerlund 1 and 2 giving a total of 60 minutes in each cluster for the blue and red spectra. In addition to the observations of the cluster fields, we measured spectrophotometric standard stars in order to derive flux calibrations. In the blue range we used LTT 4364, EG 274 and LTT 7379 (Stone \& Baldwin 1983). In the red range we added HD 160233 (Gutiérrez-Moreno et al. 1988), which is a hot dwarf star useful also to correct for telluric absorption bands. We also took frames of He-Ar comparison lamps between or after object observations, bias, dome and twilight sky and tungsten lamp flat-fields.

The reductions were carried out with the IRAF system in the standard way at the Observatorio Astronómico of the National University of Córdoba. In summary, we subtracted the bias and used flat-field frames - previously combined - to correct the frames for high and low spatial frequency variations. Then, we performed the background sky subtraction using pixel rows from the same frame, after having cleaned the background sky regions from cosmic rays. We controlled that no significant background sky residuals were present on the resulting spectra. The cluster spectra were extracted and wavelength calibrated by fitting observed He-Ar comparison lamp spectra with template spectra. The rms errors involved in these calibrations are $0.72 \AA$ ( 0.21 pixel $)$ for the blue nights and $0.39 \AA$ (0.12 pixel) for the red ones. Finally, we applied extinction corrections and flux calibrations derived from the observed standard stars to the cluster spectra. In addition, cosmic rays on the cluster spectra were eliminated. Finally, we eliminated the telluric absorption bands in the near-IR, following the procedures outlined in Bica \& Alloin (1987).

In Figs. $2 \mathrm{a}$ and $2 \mathrm{~b}$ we present the calibrated integrated spectra of Westerlund 1 and 2, respectively. Notice the very steep spectrum in the near-IR and essentially null flux shortward of $5000 \AA$ for Westerlund 1 , revealing a dramatic reddening effect.

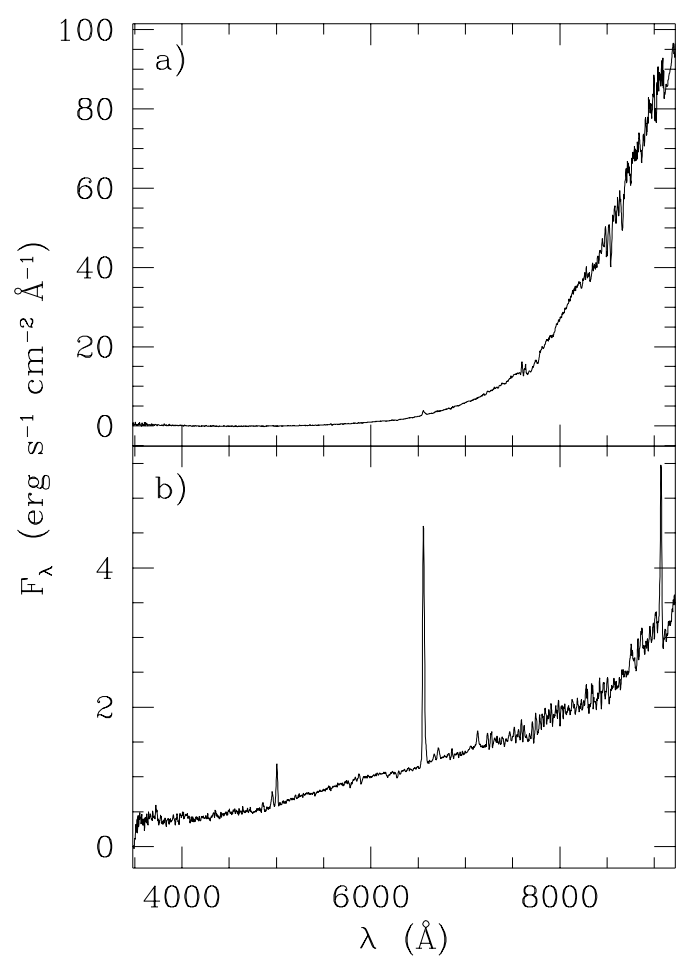

Fig. 2. Observed integrated spectra in absolute $F_{\lambda}$ units: a) Westerlund 1, b) Westerlund 2

\section{Imaging of Westerlund 1}

In Fig. 3a we show the $V$ vs. $(V-I)$ diagram for 233 measured stars in the field of Westerlund 1. It is evident that the cluster is projected against a rich field of foreground/background stars, making it very difficult to trace a clear fiducial main sequence. In order to obtain a better definition of the CMD for the cluster stars by eliminating possible non-physical members, we first determined a centre for the cluster. This was accomplished by building the stellar density profiles as a function of pixel bins in the $x$ and $y$ directions, using all stars in Fig. 3a. The final coordinates that we adopted for the position of the cluster centre are $\left(X_{\mathrm{c}}, Y_{\mathrm{c}}\right)=(300,250)$. We determined the cluster extent by calculating the average stellar density in successive 20 pixel wide annuli around the adopted centre. The resulting density profile is shown in Fig. 4, which shows that Westerlund 1 presents a core and a relatively extended low-density corona. The angular radius of the core is about $36^{\prime \prime}$ (80 pixels) while the angular cluster diameter reaches $2 ! 4$. This value was obtained by comparing the radial density profile of the cluster with independent determinations of the stellar density in four different fields around the cluster region. The derived diameter is very 

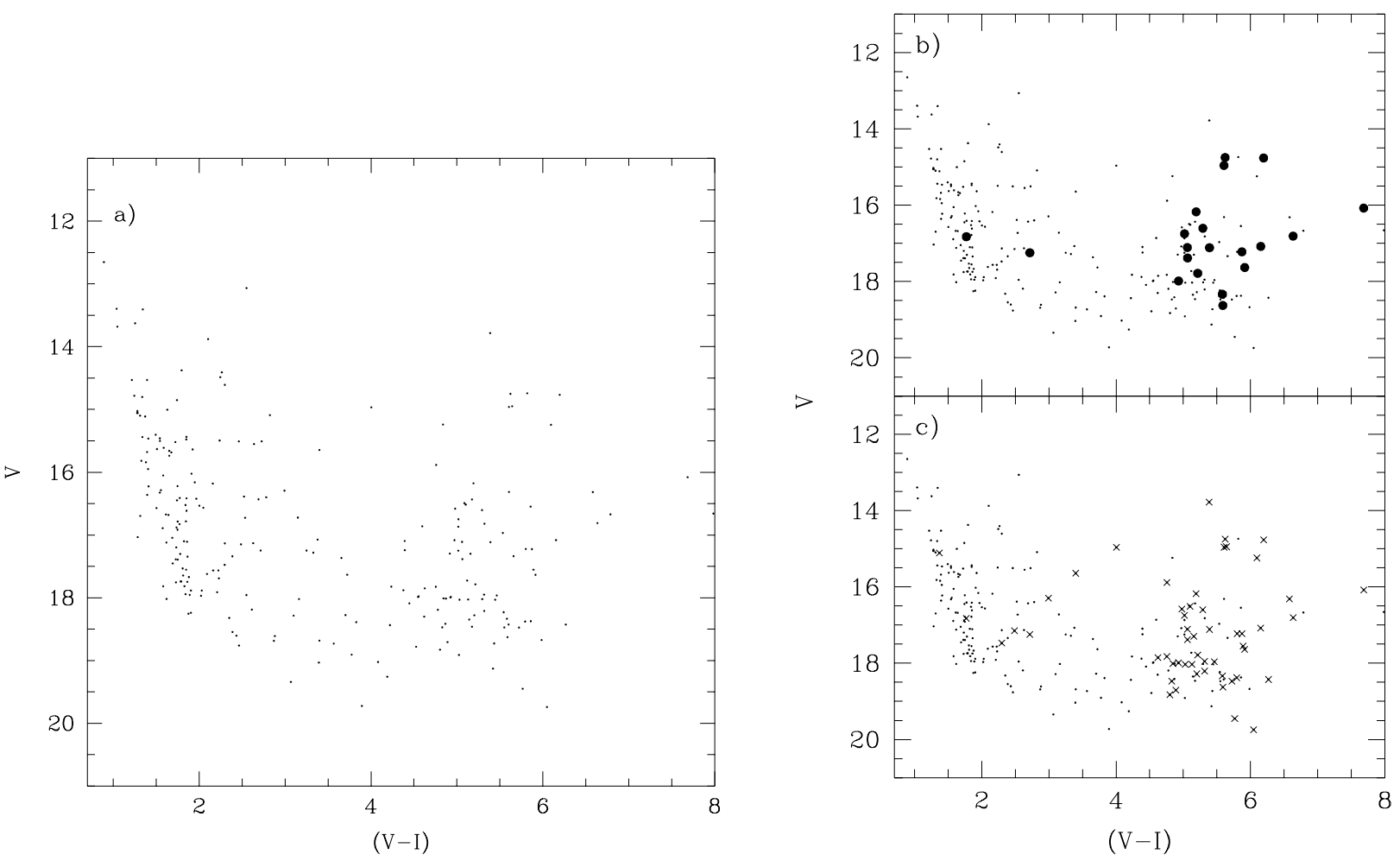

Fig. 3. Colour-magnitude diagrams for Westerlund 1: a) all measured stars (dots), b) circular extraction for $r<22$ '. 5 (filled circles) are superimposed, c) same as b) for $r<45^{\prime \prime} 0$ (crosses)

close to the average of $2^{\prime}$ and $3^{\prime}$ estimated by Westerlund (1961) and van den Bergh \& Hagen (1975), respectively.

We extracted $V$ vs. $(V-I)$ diagrams around $\left(X_{\mathrm{c}}\right.$, $\left.Y_{\mathrm{c}}\right)$ for $r<22^{\prime \prime} 5$ (50 pixels), basically corresponding to the cluster core, and $r<45^{\prime \prime} 0$ (100 pixels), thus extending to the coronal region. These diagrams are shown in Figs. 3b,c. The smallest circular extraction allowed us to know which are the CMD zones where the fiducial cluster sequences are located. Figure $3 \mathrm{c}$ is a compromise between minimizing the unavoidable field contamination and maximazing the number of cluster stars.

Despite the presence of a certain amount of field stars, the main features of the cluster CMD are now clear. Westerlund 1 appears as a vertical and very reddened main sequence affected by scatter. The vertical position of the main sequence resembles those in young open clusters. The scatter mainly arises from differential reddening, since the photometric internal errors are much smaller (Sect. 2). Figures 3b,c also exhibit several supergiant stars, as previously shown by W87.

To estimate the mean cluster reddening and apparent distance modulus we matched the CMD extractions (Figs. 3b,c) to the theoretical isochrones by Bertelli et al. (1994). Figure 5 shows the results of our fitting using a solar abundance (see Sect. 4.1) isochrone, corresponding to an age of $4 \mathrm{Myr}$; we have also included the next available isochrone (50 Myr) for comparison purposes. We derived
$E(B-V)=4.3 \pm 0.2$ or $E(V-I)=5.4 \pm 0.3$ according to the $E(V-I) / E(B-V)$ ratio given by Walker (1985), and an apparent distance modulus $V-M_{v}=23.8 \pm 0.3$. It is important to note that both the vertical position of the main sequence and its width makes it difficult to achieve an accurate placement of the isochrone, mainly along the $V$-axis. The presence of supergiants at $M_{v} \sim-9.0 \mathrm{mag}$ helped us to match the rapid evolutionary phase at the top of the $4 \mathrm{Myr}$ isochrone more properly. Using $E(B-V)=$ 4.3 and $R=A_{v} / E(B-V)=3.0$, we derived a visual absorption $A_{v}=12.9$ and a true distance modulus of $V_{0}-M_{v}=10.9 \mathrm{mag}$, equivalent to a distance of $1.5 \mathrm{kpc}$.

The derived visual absorption $A_{v}$ shows a very good agreement with the values found by Lockwood (1974) from stars observed at near-infrared wavelengths $(0.71-1.05 \mu)$, and that of Borgman et al. (1970) using $K$-filter observations. We have also obtained a similar value of $A_{v}$ from our CCD integrated spectrum (see Sect. 4.1). The present visual absorption and true distance modulus differ significantly from the values derived by W87, whereas they are in fairly good agreement with Westerlund's (1961) earlier results (see also Sect. 1).

Looking for a possible explanation for the difference with W87, we first examined how similar both (CCD and photographic) photometries are. This was done from $8 \mathrm{su}-$ pergiants spread out within the cluster core region and for which W87 obtained MK spectral-types, $V$-Johnson 


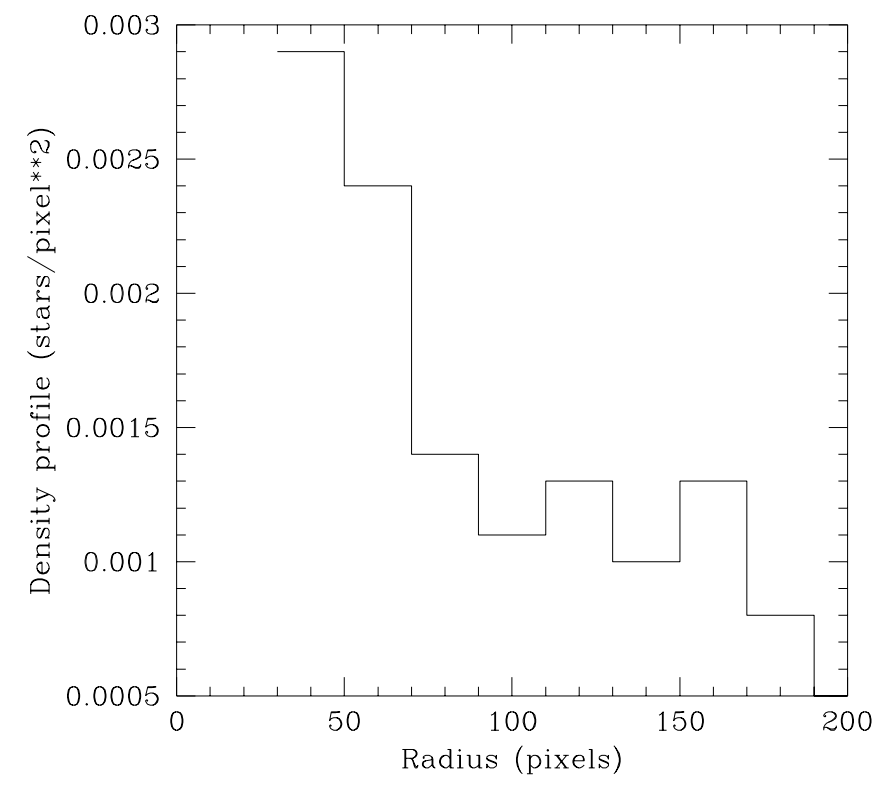

Fig. 4. Density profile of Westerlund 1

magnitudes and $(V-I)$ Kron colour indices. The comparison between both $V$ magnitude scales yields a mean difference of $0.3 \pm 0.07 \mathrm{mag}$, our values being sistematically brighter. To compare the $(V-I)$ colour indices we first transformed the present $(V-I)$ Cousins values to the Kron system. For this purpose, we used the MK vs. $(V-I)_{\mathrm{K}}$ relationship given by W87, Bessell's (1979) relations between the Cousins and Kron systems, and the fact that the ratio $A_{v} / E(V-I)$ is the same in both systems (W87). A comparison of our transformed $(V-I)$ indices with the values published by W87 yields a mean difference of $0.8 \mathrm{mag}$. This implies a $0.8 \mathrm{mag}$ shift in the $E(V-I)$ colour excess, which in turn leads to an $A_{v}$ value increased by $1.6 \mathrm{mag}$ with respect to that of W87. Therefore, taking into account these zero points, the revised W87 true distance modulus and cluster distance are 11.7 and about $2.0 \mathrm{kpc}$, respectively, which are now consistent with the present CMD-fitting values.

An alternative way to derive the cluster distance is to use our CCD $V$ magnitudes and $M_{v}$ from W87 for the nine brightest stars in the cluster central region. We obtained $V-M_{v}=22.9 \pm 0.7$, equivalent to a distance of $1.2_{-0.2}^{+0.3} \mathrm{kpc}$, adopting $A_{v}=12.5 \pm 0.2$ computed from appropriate $R$ values (Crawford \& Mandwewala 1976). This distance is somewhat smaller than that we obtained from the fitting analysis (Fig. 5). However, if the couple $\left(A_{v}\right.$, $\left.V-M_{v}\right)=(12.9,22.9)$ is adopted, the $4 \mathrm{Myr}$ isochrone in Fig. 5 would be displaced downwards nearly $1.0 \mathrm{mag}$, the fit being still satisfactory. In this case, only the brightest star (G0Ia+, No. 4 in W87) would slightly fall away from the sequence. Nevertheless, it might still be a member considering the amount of differential reddening present in the cluster.

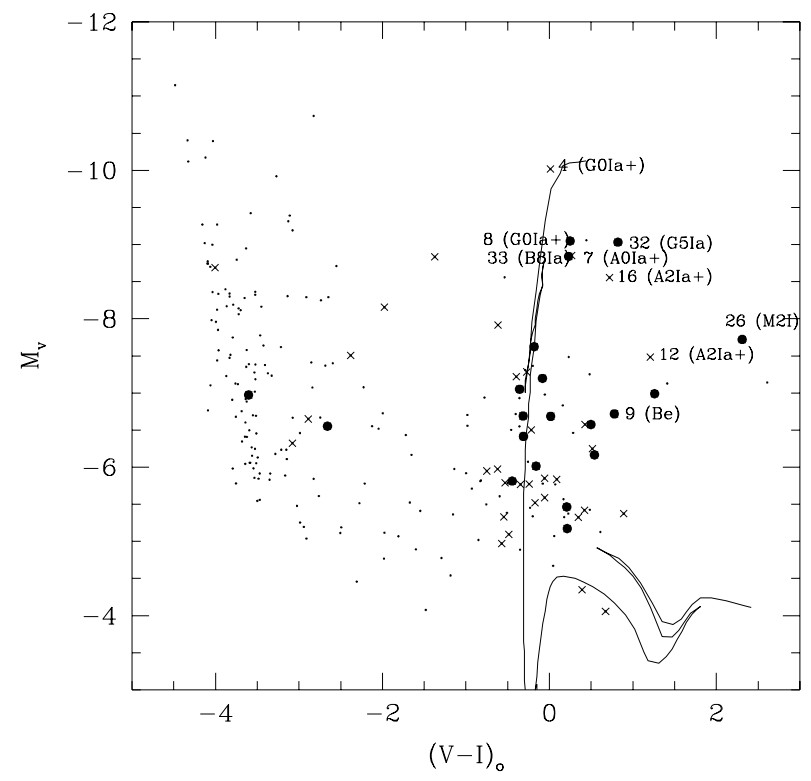

Fig. 5. HR diagram of Westerlund 1 . The solid lines are the solar metallicity isochrones of Bertelli et al. (1994) corresponding to 4 and $50 \mathrm{Myr}$, respectively. Symbols are as in Fig. 3. Identification and spectral types of stars are from Westerlund (1987)

An additional method to derive distance is to use the $M_{v}$ magnitudes given by Mermilliod (1981a,b) from his open cluster age groups. We have recognized several features in the $V$ vs. $(V-I)$ plane with the aid of W87's MK spectral types, which resemble those in Mermilliod's NGC 457 and NGC 884 age groups $(10-15 \mathrm{Myr})$. These features are: (i) a concentration of blue and yellow supergiants (Nos. 7, 8, 16, 32 and 33 in W87), (ii) a single very bright blue supergiant (No. 4), (iii) a red supergiant (No. 26, M2I), (iv) a Be star (No. 9), (v) the upper main sequence, and (vi) a $V$ gap between the main sequence and the hotter supergiants. These features are consistent with a cluster distance of about $0.7 \mathrm{kpc}$.

After considering the three independent determinations, we adopted $d=1.1 \pm 0.4 \mathrm{kpc}$, thus considerably improving the knowledge of the distance for Westerlund 1.

\section{Integrated spectroscopy}

Integrated spectra of star clusters allow one to determine their basic parameters such as reddening, age and metallicity. Bica \& Alloin $(1986,1987)$ have studied integrated spectra in the visible and near-infrared ranges of Galactic open and globular clusters, as well as Magellanic Cloud clusters. They investigated the behaviour of metallic and Balmer-line equivalent widths $(W)$ as well as the continuum energy distribution from 3700 to $10000 \AA$. Once the age and metallicity are established from $W$ measurements and comparisons with templates, the reddening can be 
derived by matching the observed cluster spectrum to that of the template which most resembles it.

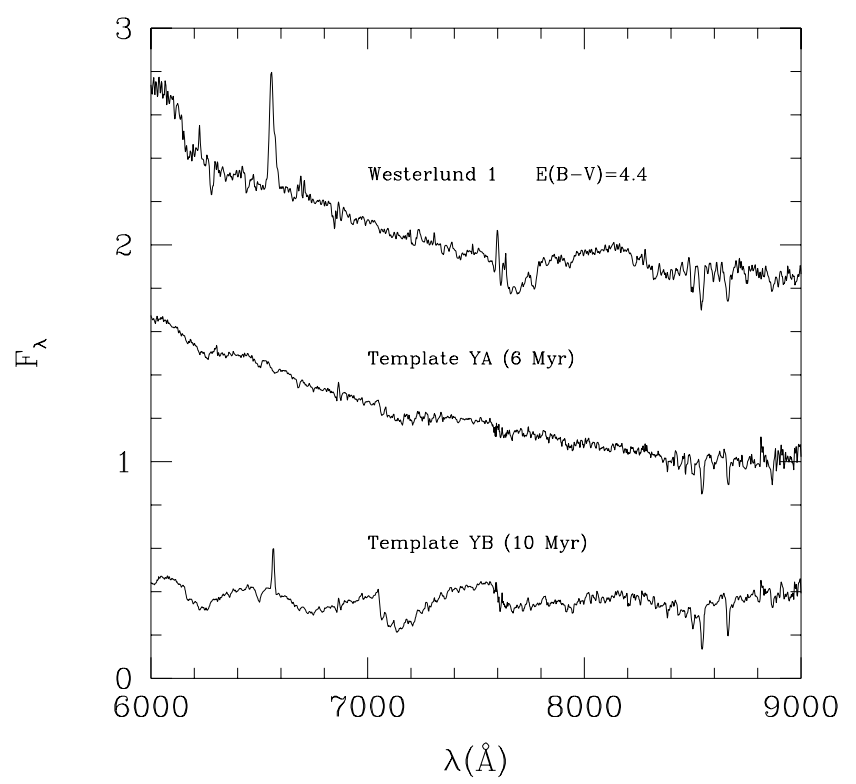

Fig. 6. Integrated spectrum of Westerlund 1 corrected for $E(B-V)=4.4($ top $)$, the template YA (middle) and the template YB (bottom)

\subsection{Westerlund 1}

We show in Fig. 6 the near-IR integrated spectrum of Westerlund 1, corrected for $E(B-V)=4.4$ (see discussion below). Given the extremely high absorption for Westerlund 1, its spectrum has no measurable flux for $\lambda \lesssim 5000 \AA$. The prominent $\mathrm{H} \alpha$ could arise either from stellar sources in clusters younger than $35 \mathrm{Myr}$ (Bica et al. 1990), or extended gas (e.g. HII region). As already pointed out by W87 star No. 9 is a Be star with pronounced emission lines, and star No. 31 is possibly another Be. W87 also detected diffuse $\mathrm{H} \alpha$ emission in the cluster central region. We have investigated in our integrated spectrum the intensity variation of $\mathrm{H} \alpha$ across the cluster profile and found that the highest values occur within a small region approximately 5 pixel wide, in the central part of the cluster. The emission is fainter in the remaining zones of the central region as well as in the wings of the cluster profile. The pronounced inner $\mathrm{H} \alpha$ emission could be assigned to the Be star located in the central part of the cluster (see Fig. 1 in W87), while the weak emission could be due to extended nebular gas. Diffuse emission occurs in HII regions as old as $5 \mathrm{Myr}$, or even older clusters which have residual gas emission from photoionization and/or supernova remnants, as can be seen in the templates of Santos et al. (1995).

The presence of $\mathrm{H} \alpha$ in emission, when caused by stellar sources such as Be stars, is a remarkable feature in the YB and YC templates of Bica et al. (1990), whose age ranges are $7-12 \mathrm{Myr}$ and $12-35 \mathrm{Myr}$, respectively. The YB template reflects the full development of the red supergiant (RSG) phase, and consequently the near-IR spectrum presents strong $\mathrm{TiO}$ bands and $\mathrm{CaII}$ triplet lines as well as a flat continuum. We show in Fig. 6 the YB template, where the strong $\mathrm{H} \alpha$ emission and the effects of the RSGs can be seen. The slightly older YC template (see Bica et al. 1990) is bluer, since RSGs do not contribute significantly.

The M2I star (No. 26 in W87) near the center of Westerlund 1 produces moderate $\mathrm{TiO}$ bands around $\lambda 6300 \AA$ and $\lambda 8300 \AA$ in our reddening-corrected integrated spectrum (Fig. 6). Notice also that the CaII triplet is well developed in the cluster, which sets a lower age limit of 5 Myr; the template YA in Bica et al. (1990), corresponding to this evolutionary stage $(5 \mathrm{Myr} \leq$ age $\leq$ $7 \mathrm{Myr}$ ), is also shown in Fig. 6.

The above considerations about occurrence of diffuse and stellar emissions, RSG, and developed CaII triplet indicate that the most probable age for the cluster is $8 \pm$ $3 \mathrm{Myr}$, in very good agreement with the estimation of $7-$ 9 Myr by W87.

A prominent absorption feature in the range $\lambda \lambda 7550-$ $7850 \AA$ is present. Sanner et al. (1978) have studied several diffuse interstellar features in the near-IR, including four within this range. Based on integrated spectra of globular clusters, Armandroff \& Zinn (1988) have studied an interstellar feature at a longer wavelength $(\lambda 8621 \AA)$, clearly detectable for $E(B-V)>1.2$. We suggest that the absorption feature found in the $\lambda \lambda 7550-7850 \AA$ region is of interstellar origin, because it is also prominent in globular clusters more reddened than $E(B-V)=2.0$ (Bica et al. 1997). Sanner et al. (1978) have only detected a few interstellar lines in this region, probably due to the fact that their stars are not enough reddened, and also because their spectra were not corrected for the telluric A band.

The reddening of Westerlund 1 was obtained by varying $E(B-V)$ following Seaton's (1979) law to match the continuum of the YA template, as shown in Fig. 6. Notice the flux excess of the cluster spectrum with respect to that of the YA template for $\lambda>8000 \AA$, which we attribute to the contribution of the M2I star. An attempt to match the RSG-dominated continuum of the YB template yields $E(B-V)=4.0$. We favour the higher reddening value because the cluster spectrum resembles most that of the YA template. This spectroscopically derived reddening is consistent with that estimated from the CMD-fitting (Sect. 3). We finally adopted $E(B-V)=4.3 \pm 0.2$.

We measured $W$ of the CaII triplet lines using as continuum points the regions around $\lambda=8408 \AA$ and $\lambda=8700 \AA$ (Bica \& Alloin 1987). Table 2 presents the window limits and the $W \mathrm{~s}$. The error assigned to each measurement was calculated by considering local high and low continuum tracings in order to take into account the 
spectral noise. Although the correlations between $W$ of CaII triplet lines with metallicity are basically singlevalued for integrated spectra of star clusters (Bica \& Alloin

1987), there are sources of scatter which affect the $\left[Z / Z_{\odot}\right]$ determination. These uncertainties are related to the different stellar components contributing to the integrated light rather than to the errors arising from the quality of the spectrum itself. The contamination by $\mathrm{TiO}$ bands and Paschen lines affects the CaII windows. TiO may reinforce the metallicity dependence of the CaII triplet (especially for the 8498 and $8542 \AA$ lines), while Paschen lines introduce a systematic increase for blue clusters whatever their metallicity. The Paschen absorptions affect more the CaII $8662 \AA$ line.

Table 2. Equivalent widths $(\AA)$

\begin{tabular}{lll}
\hline Window & Limits $(\AA)$ & Westerlund 1 \\
\hline $\mathrm{CaII}+\mathrm{TiO}$ & $8476-8520$ & $2.20 \pm 0.18$ \\
$\mathrm{CaII}+\mathrm{TiO}$ & $8520-8564$ & $4.77 \pm 0.13$ \\
$\mathrm{CaII}+\mathrm{P} 13$ & $8564-8700$ & $5.52 \pm 0.04$ \\
\hline
\end{tabular}

To estimate the metallicity of Westerlund 1 we compared the $W(\mathrm{CaII})$ values with those measured by Santos \& Bica (1993) and Bica \& Alloin (1987) for three open clusters with independent metallicity determinations from individual stars. The sum of the equivalent widths for the three CaII lines in Westerlund 1 is $\Sigma W=12.5 \pm 0.4$. This value compares well with the average $(\Sigma W=11.0 \pm$ 1.9) of those for NGC 4755, NGC 6067, and NGC 6705, whose $[\mathrm{Fe} / \mathrm{H}]$ values are 0.0 (Brown et al. 1986), -0.1 (Piatti et al. 1995) and +0.21 (Thogersen et al. 1993), respectively. We thus conclude that Westerlund 1 has nearly solar metal content.

\subsection{Westerlund2}

The observed CCD integrated spectrum of Westerlund 2 (Fig. 2b) was obtained by scanning the slit $\sim 25^{\prime \prime}$ across the central region of the cluster in the north-south direction. The most relevant features in this spectrum are the nebular emission lines [OIII] $4959,5007 \AA$, $\mathrm{H} \alpha$, and [SIII] $9068 \AA$, and a continuum with a pronounced slope denoting important reddening. These nebular emission lines are expected since Westerlund 2 is the core of the HII region RCW 49 (Belloni \& Mereghetti 1994). We adopted as template spectrum that of the cluster NGC 3603, which is also the core of an HII region (Santos \& Bica 1993 and references therein). We assume as the age of Westerlund 2 that of NGC 3603, i.e., $2-3$ Myr (Melnick et al. 1989; Santos \& Bica 1993). This value is consistent with the presence of a WN7 star (MSP91) located away from the core of Westerlund 2, not included in our integrated spectrum. Furthermore, MSP91 have spectroscopically recognized several O7V stars, which confirms the adopted age range.

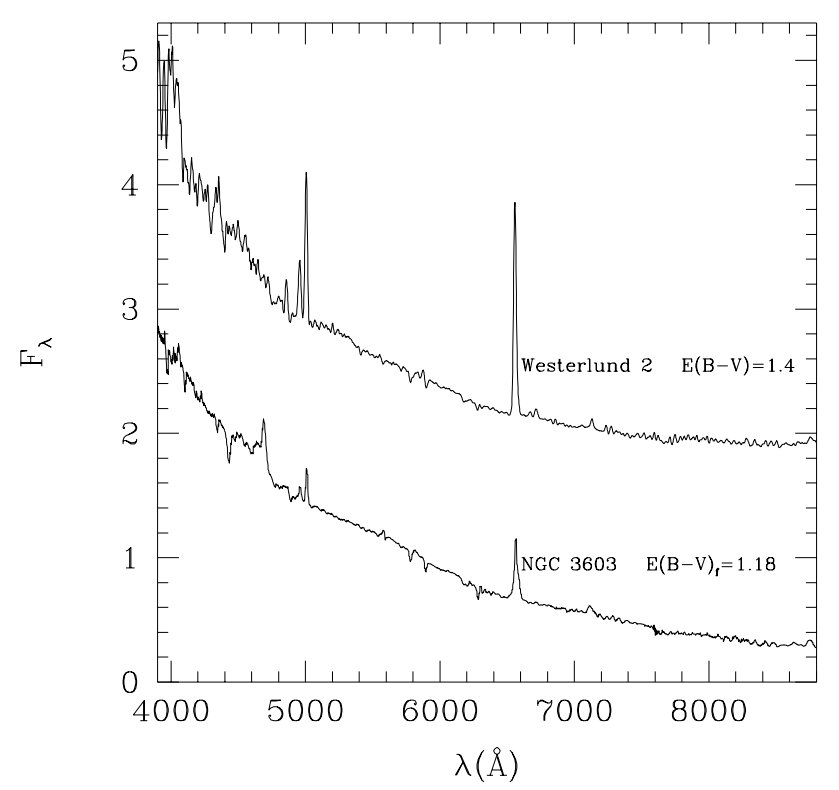

Fig. 7. Integrated spectrum of Westerlund 2 corrected for $E(B-V)=1.4$ (top) compared to the NGC 3603 corrected for foreground $E(B-V)_{\mathrm{f}}=1.18$ (bottom)

We show in Fig. 7 the spectrum of Westerlund 2 with a reddening correction $E(B-V)=1.4$ to match that of NGC 3603, which in turn is corrected for its foreground reddening $\left(E(B-V)_{\mathrm{f}}=1.18\right.$, Santos \& Bica 1993). Notice that the core of NGC 3603 presents several WR stars (Moffat \& Niemela 1984), which are responsible for the observed WN features in the integrated spectrum. The CaII triplet has not yet developed in such young cluster, and consequently we have not measured equivalent widths.

Our spectroscopic reddening estimation should be considered as a foreground value. From 72 member stars of Westerlund 2 in MSP91's Table 2, we derived a mean cluster reddening of $E(B-V)=1.67 \pm 0.15$. The difference between this value and the spectroscopic one suggests an internal reddening of $\Delta E(B-V)=0.27$ in such a young object. For comparison, Santos \& Bica (1993) estimated an internal reddening of $\Delta E(B-V)=0.33$ in NGC 3603.

We have reexaminated MSP91's estimation of the cluster distance $(7.9 \mathrm{kpc})$ by considering four cluster core O7V stars (Nos. 167, 183, 188 and 203). We derived from their $V$ and $M_{v}$ magnitudes a mean apparent distance modulus of $18.79 \pm 0.38$. Subsequently, using the information available in MSP91, the mean $E(B-V)$ colour excess for these stars was determined in two different ways. First, we compared the observed CCD $(B-V)$ index with the 
intrinsic $(B-V)_{0}$ colour according to the MK type. The second method consists simply in averaging the $E(B-V)$ values calculated by MSP91 from their $U B V$ photometry. These procedures yield $E(B-V)=1.70 \pm 0.06$ and $1.64 \pm 0.02$, respectively, which in turn lead to a cluster distance of 5.5 and $5.9 \mathrm{kpc}$. The considerably larger distance obtained by MSP91 certainly comes from uncertainties in their ZAMS-fitting, owing to the almost vertical position of the observed CMD-sequence.

\section{The structure of Westerlund 1}

In order to investigate the structure of Westerlund 1 , we compare its intrinsic size with those of the compact young open clusters NGC 3603 and Westerlund 2. For the sake of uniformity, we measured their angular dimensions on the ESO/SRC Schmidt plates, obtaining: $2.8 \times 2 ! 8$, 1 '. $45 \times 1$ '.0 and 1 '.35 $\times 1$ 1'0 for Westerlund 1 , Westerlund 2 and NGC 3603, respectively. Using the distances of $1.1 \mathrm{kpc}$ (Sect. 3), $5.7 \mathrm{kpc}$ (Sect. 4.2) and $7.2 \mathrm{kpc}$ (Melnick et al. 1989), the corresponding linear sizes are: $0.8 \mathrm{pc} \times 0.8 \mathrm{pc}$, $2.4 \mathrm{pc} \times 1.6 \mathrm{pc}$ and $2.8 \mathrm{pc} \times 2.1 \mathrm{pc}$, respectively. Therefore, the size of Westerlund 1 is comparable to those of the massive clusters NGC 3603 and Westerlund 2. Recently, Watson et al. (1996) have examined the central region of the starburst spiral galaxy NGC 253 using high resolution imaging. They have reported the discovery of four compact young luminous stellar clusters, with half-light radii between 1.0 and $5.0 \mathrm{pc}$, and masses and light profiles comparable to those of Galactic globular clusters. The existence of such clusters in starburst galaxies suggests that enhanced star formation processes are required to form them.

We also measured on the ESO/SRC Schmidt plates the angular dimensions of Gum 29 (Gum 1955), which is part of the HII region RCW 49 (Rodgers et al. 1960) directly associated to Westerlund 2, and obtained $11^{\prime} .4 \times 10 ! 3(4 ! .8 \times$ 4 ' 4 for the bright core). For the HII region associated to NGC 3603 (Gum 38b, which in turn is the eastern part of RCW 57), we obtained 10!. $\times 8.9(7.3 \times 4.7$ for the bright core). The linear sizes of Gum 29 give $18.9 \mathrm{pc} \times 17.1 \mathrm{pc}$ $(7.9 \mathrm{pc} \times 7.2 \mathrm{pc}$ for the core), and those of Gum $38 \mathrm{~b}$, $22.0 \mathrm{pc} \times 18.6 \mathrm{pc}(15.3 \mathrm{pc} \times 9.8 \mathrm{pc}$ for the core $)$. Assuming similar gas densities, this suggests that the bulk of the ionization caused by Westerlund 2 is comparable to that of NGC 3603, showing that we are also dealing with a massive star cluster. No HII region in the Gum nor in the RCW catalogues appears to be related to Westerlund 1, nor is any apparent on the ESO/SERC R Schmidt plate. Westerlund 1 is somewhat older than the former clusters (Sects. 3, 4.1 and 4.2), but still presents residual nebular emission lines in its spectrum (Sect. 4.1).

Finally, we study the radial light distribution of Westerlund 1 from our CCD $V$ image. We used the magnitude of individual stars (Sect. 2) and integrated them in radial annuli. In addition, we considered circles with

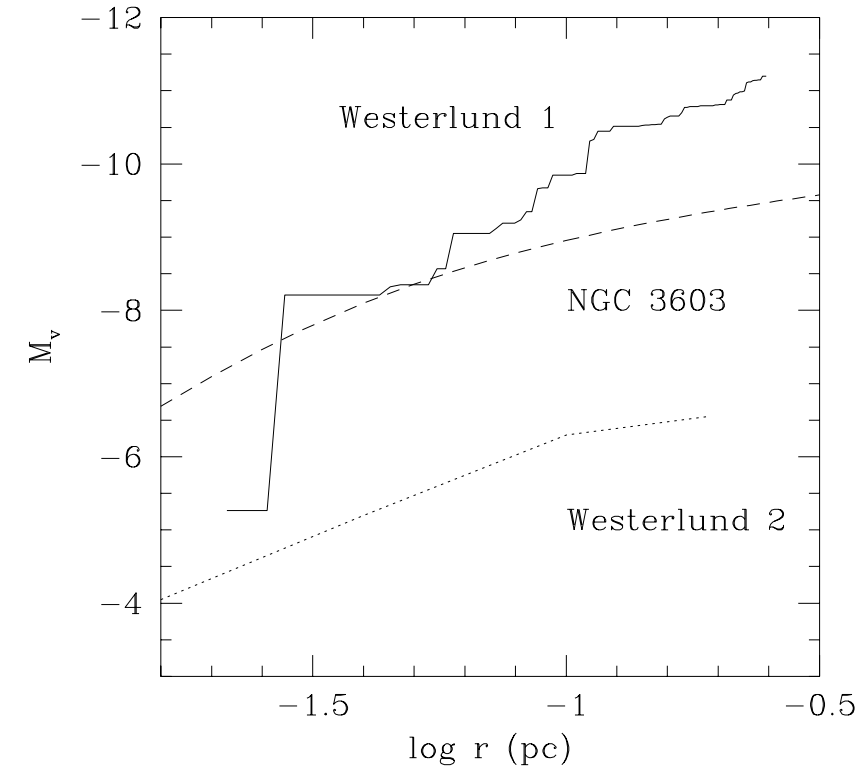

Fig. 8. Radial light distribution for NGC 3603 (dashed line), Westerlund 2 (dotted line) and Westerlund 1 (solid line)

radii smaller than 100 pixels, thus including the core and part of the corona, to minimize the field contamination (see Sect. 3). We compare in Fig. 8 the resulting radial light distribution to those of NGC 3603 and Westerlund 2 (MSP91), in terms of the accumulated absolute visual magnitude as a function of radius. We adopted our distance estimation for Westerlund 2, which placed the cluster 0.7 mag fainter than in MSP91. Since the clusters have similar ages, we conclude, from the accumulated $M_{v}$ at the larger radius available, that Westerlund 1 is as massive as NGC 3603. Although we placed Westerlund 2 closer with respect to MSP91, it is still a rather massive open cluster. Finally, contrarily to what has been pointed out by MSP91, NGC 3603 is not the unique case of massive cluster in the Galactic disk, comparable to the 30 Dor cluster in the LMC and the four massive young clusters in the starburst galaxy NGC 253 (Watson et al. 1996).

As shown by Moffat et al. (1985) the central parts of NGC 3603, having a compact small core, are well fitted by a King's (1962) profile. Westerlund 2 may have a similar feature, but could not be resolved in MSP91. For Westerlund 1, we could not satisfactorily fit a King profile with reasonable $r_{\mathrm{c}}$ values. A fundamental question is how large amounts of luminous stars, confined in a relatively small volume, could be formed either in massive clusters as NGC 3603, or in small compact open clusters as Westerlund 1 (which has approximately 15 luminous stars within $r=0.1 \mathrm{pc}$ )? Efforts in this direction are, e.g., the numerical simulations by Murray \& Lin (1996). They found that the most massive stars require many dissipative cloudlet mergers, and so are preferentially formed in the cluster center. 


\section{Concluding remarks}

We have presented new CCD VI photometry for Westerlund 1 and integrated spectra in the visible and near-IR ranges for Westerlund 1 and 2. The analysis of the observed data allowed us to improve the knowledge of the fundamental properties for both clusters. Westerlund 1 is a compact open cluster with an age of $8 \pm 3$ Myr located at $1.1 \pm 0.4 \mathrm{kpc}$ from the sun. It is affected by a strong interstellar visual absorption $\left(A_{v}=12.9 \mathrm{mag}\right)$, which, as far as we aware, converts it into the most reddened open cluster in the Galaxy studied in detail. From the CaII triplet lines we conclude that the cluster has a nearly solar metallicity. Westerlund 1 is a massive cluster, but it contains several luminous stars confined in a relatively small volume.

For Westerlund 2 we estimated an age of $2-3 \mathrm{Myr}$, a foreground reddening $E(B-V)_{\mathrm{f}}=1.4$ together with evidence of some amount of internal reddening. We derived a revised distance for Westerlund 2 of $5.7 \pm 0.3 \mathrm{kpc}$, with the help of complementary observations taken from the literature. Westerlund 2 is quite massive, but not as much as NGC 3603.

The formation of massive globular-like clusters apparently requires much larger star-forming complexes than are now usually found in the Milky Way; they form in the densest parts of such complexes, and the molecular clumps in which they are embedded are compressed in part by the effects of earlier star formation (Larson 1993). NGC 3603 possibly satisfies those conditions. The concentration of supergiants in the central region of Westerlund 1 is due to a somewhat smaller scale enhanced star formation event.

Acknowledgements. We acknowledge use of the CCD and data acquisition system at CASLEO supported under U.S. National Science Foundation grant AST-90-15827 to R.M. Rich. We are grateful to the staff at CASLEO and Las Campanas, for their kind hospitality and assistance during the observing runs. We are also very indebted to the referee Dr. J.C. Mermilliod whose valuable comments and suggestions helped us to improve the manuscript. This work was partially supported by the institutions CNPq and FINEP (Brazil), and CONICET and CONICOR (Argentina). We also acknowledge support from the Vitae and Antorchas foundations.

\section{References}

Alter G., Balázs B., Ruprecht J., 1970, Catalogue of Star Clusters and Associations, 2nd ed., Budapest, Akademia Kiadó

Armandroff T.E., Zinn R., 1988, AJ 96, 92

Belloni T., Mereghetti S., 1994, A\&A 286, 935

Bertelli G., Bressan A., Chiosi C., Fagotto F., Nasi E., 1994, A\&AS 106, 275
Bessell M.S., 1979, PASP 91, 589

Bica E., 1988, A\&A 195, 76

Bica E., Alloin D., 1986, A\&A 162, 21

Bica E., Alloin D., 1987, A\&A 186, 49

Bica E., Alloin D., Santos Jr. J.F.C., 1990, A\&A 235, 103

Bica E., Clariá J.J., Piatti A.E., Bonatto C., 1997 (in preparation)

Borgman J., Koornneef J., Slingerland J., 1970, A\&A 4, 248

Brown P.J.F., Dufton P.L., Lennon D.J., Keenan F.P., 1986, MNRAS 220, 1003

Crawford D.L., Mandwewala N., 1976, PASP 88, 917

Gascoigne S.C.B., Kron G., 1952, PASP 64, 196

Gum C.S., 1955, Mem. R. Astron. Soc. 67, 155

Gutiérrez-Moreno A., Moreno H., Cortés G., Wenderoth E., 1988, PASP 100, 973

Hodge P.W., 1961, ApJ 133, 413

Holmberg E.B., Lauberts A., Schuster H.-E., West R.M., 1977, A\&AS 27, 295

King I.R., 1962, AJ 67, 471

Landolt A.U., 1992, AJ 104, 340

Larson R.B., 1993, The globular cluster - galaxy connection. In: Smith G.H., Brodie J.P. (eds.) Proc. XI Santa Cruz Summer Workshop in Astronomy and Astrophysics, p. 673 Lockwood G.W., 1974, ApJ 193, 103

Melnick J., Tapia M., Terlevich R., 1989, A\&A 213, 89

Mermilliod J.C., 1981a, A\&A 97, 235

Mermilliod J.C., 1981b. In: Jaschek M., Hans-Günter Groth (eds.) Proc. IAU Symp. 98, Be Stars. Dordrecht, p. 23

Moffat A.F.J., 1983, A\&A 124, 273

Moffat A.F.J., Niemela V.S., 1984, ApJ 284, 631

Moffat A.F.J., Seggewiss W., Shara M.M., 1985, ApJ 295, 109

Moffat A.F.J., Shara M.M., Potter M., 1991, AJ 102, 642 (MSP91)

Murray S.D., Lin D.N., 1996, ApJ 467, 728

Piatti A.E., Clariá J.J., Abadi M.G., 1995, AJ 110, 2813

Price S.D., 1968, AJ 73, 431

Rodgers A.W., Campbell C.T., Whiteoak J.B., 1960, MNRAS 121,103

Sanner F., Snell R., Vanden Bout P., 1978, ApJ 226, 460

Santos Jr. J.F.C., Bica E., 1993, MNRAS 260, 915

Santos Jr. J.F.C., Bica E., Clariá J.J., et al., 1995, MNRAS 276,1155

Seaton M.J., 1979, MNRAS 187, 73

Stetson P.B., 1991, DAOPHOT User Manual

Stone R.P.S., Baldwin J.A., 1983, MNRAS 204, 347

Thogersen E.N., Friel E.D., Fallon B.V., 1993, PASP 105, 1253

van den Bergh S., Hagen G.L., 1975, AJ 80, 11

Walker A.R., 1985, MNRAS 213, 889

Watson A.M., Gallagher III J.S., Holtzman J.A., et al., 1996, AJ 112, 534

Westerlund B.E., 1960, Ark. Astron. 2, 419

Westerlund B.E., 1961, PASP 73, 51

Westerlund B.E., 1968, ApJ 154, L67

Westerlund B.E., 1987, A\&AS 70, 311 (W87) 